\title{
Strong magnetic field dependence of critical current densities and vortex activation energies in an anisotropic clean $\mathrm{MgB}_{2}$ thin film
} \author{
Movshovich $^{5}$ and L. Civale. ${ }^{5}$ \\ ${ }^{1}$ CALDES, Institute for Basic Science, Pohang, Korea \\ ${ }^{2}$ Department of Physics, Pohang University of Science and Technology, Pohang, Korea \\ ${ }^{3}$ Centro Atómico Bariloche, Bariloche, 8400, Argentina. \\ ${ }^{4}$ Brookhaven National Laboratory, Upton, NY 11973, USA. \\ ${ }^{5}$ Los Alamos National Laboratory, Los Alamos, NM 87545 USA \\ ${ }^{6}$ Department of Physics, Temple University, Philadelphia, PA 19122, USA.
}

Jeehoon Kim, ${ }^{1,2}$ N. Haberkorn, ${ }^{3}$ E. Nazaretski, ${ }^{4}$ R. de Paula, ${ }^{5}$ Teng Tan, ${ }^{6}$ X. X. Xi, ${ }^{6}$ T. Tajima, ${ }^{5}$ R.

We report the influence of two-band superconductivity on the flux creep and the critical current

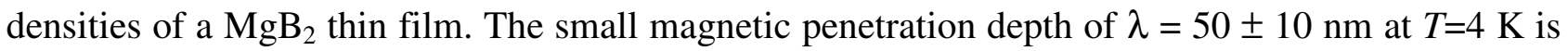
related to a clean $\pi$-band. We find a high self-field critical current density $J_{c}$, which is strongly reduced with applied magnetic field, and attribute this to suppression of the superconductivity in the $\pi$-band. The temperature dependence of the creep rate $S(T)$ at low magnetic field can be explained by a simple Anderson-Kim mechanism. The system shows high pinning energies at low field that are strongly suppressed by high field.

Corresponding author at: CALDES, Institute for Basic Science, Pohang, Republic of Korea, Dpartment of Physics, Pohang University of Science and Technology, Pohang, Korea. Tel.: +82 54 279 2077; fax: + 8254279 9889. E-mail addresse ${ }^{\text {s: jeehoon@ postech.ac.kr (J. Kim) }}$

Keywords:

A. Superconducting materials;

B. Films;

C. Magnetic penetration depth;

D. Vortex pinning;

\section{Introduction}

The binary $\mathrm{MgB}_{2}$ compound has been subject of intense studies since the discovery of its superconductivity. i,ii The two-band superconductivity consists of two independent electronic bands, connected by inter-band coupling via impurity scattering, and hence different resulting properties. ${ }^{\text {iii,iv,v }}$ The $\mathrm{MgB}_{2}$ system presents two distinct $s$-wave superconducting gaps - from the two dimensional (2D) $\sigma$ band and the three dimensional (3D) $\pi$ band, with superconducting energy gaps of $\Delta_{\sigma}(0) \approx 7.2 \mathrm{mV}$ and $\Delta_{\pi}(0) \approx 2.3 \mathrm{mV}$, which leads to both inter- and intra-band scattering, and 
results in a rich array of superconducting properties. ${ }^{\text {ii,iv }}$ The strong suppression of the $\pi$-band superconductivity with increasing magnetic field in clean systems can be inferred from differences between the upper critical field anisotropy $\left(\gamma_{H c 2}=H_{c 2}^{a b} / H_{c 2}^{c}=\xi_{a b} / \xi_{c}\right)^{\text {vi }}$ and the magnetic penetration depth anisotropy $\left(\gamma_{\lambda}=\lambda_{a b} / \lambda_{c}\right) \cdot{ }^{\text {vii, viii,ix }}$ The difference of the magnetic field dependence of the two gaps is also manifested in the Ginzburg-Landau parameter $k=\lambda / \xi$, which ranges from $k=2-3$ at low field to $k=7$ close to $H_{c 2}{ }^{\mathrm{x}}$ In dirty samples disorder (via several mechanisms) affects the intraband diffusivity in each band, and thereby the resulting physical properties. ${ }^{\text {ii }}$ Recently we reported the influence of large intra-band diffusivity in the 3D $\pi$ band on the superconducting properties in a $\mathrm{MgB}_{2}$ thin film in the dirty limit, ${ }^{\mathrm{xi}}$ which results in larger $\lambda$ and smaller $\gamma_{H c 2}$ than those found in clean single crystals. ${ }^{\mathrm{x}}$ The modification of the superconducting properties in a multiband system provides a venue for both basic and applied point of view. For example, a change in interband coupling strength is suggested to affect the $\xi(T)$ behavior related to the weak band. In addition to the ordinary critical temperature $T_{c}$, a hidden critical point at the critical temperature of the weaker band in the absence of coupling is expected. ${ }^{\text {xii }}$ On the other hand, technological applications such as magnets require high upper critical fields, ${ }^{\text {iv }}$ and Josephson junctions require high band gap. ${ }^{\text {xii }}$

The unconventional superconducting properties in $\mathrm{MgB}_{2}$ allow the investigation of the influence of the two superconducting gaps on the flux creep. The strong suppression of the superconductivity in the $\pi$ band with magnetic field $H$ (below $1 \mathrm{~T}$ ), is directly related to the effect of the changing $\lambda$ on the depairing critical current $\left(J_{0}\right),{ }^{\mathrm{x}}$ and should influence $J_{c}(H)$ in a way that goes beyond the type of pinning centers alone. The $J_{0}$ values in $\mathrm{MgB}_{2}$ depend on the microscopic parameters of the two band involved, such as inter- and intra-band coupling strength, density of states at the Fermi surface and Fermi velocity for each band. ${ }^{\text {xiv }}$ In general, in the context of defects as pining centers, clean single crystals show low $J_{c}$ values, ${ }^{\mathrm{xv}}$, ${ }^{\text {vvi }}$ while thin films show higher $J_{c}$ values. ${ }^{\text {vii,xviii }}$ The vortex dynamics in dirty $\mathrm{MgB}_{2}$ films is characterized by low creep rates $(S)$ and high pinning energies, ${ }^{\text {xix }}$ a behavior intermediate between the low $T_{c}$ superconductors and the high $T_{c}$ cuprates. ${ }^{\mathrm{xx}}$ In addition, the high $J_{c}$ values reported in clean $\mathrm{MgB}_{2}$ films at low $H,{ }^{\mathrm{v}}$ indicating that the nature of pinning is due to a combination of both intrinsic superconducting properties and pinning landscape.

We report the intrinsic superconducting properties and their influence on the flux creep in a $\mathrm{MgB}_{2}$ thin film. We have directly determined the $\lambda$ value, the upper critical fields, as well its angular dependence was determined by electrical transport measurements. The critical currents $\left(J_{c}\right)$ and the vortex pinning energy $\left(U_{0}\right)$ are strongly affected by magnetic field, which can be associated with the magnetic field suppression of superconductivity in the clean $\pi$-band. These results are different to those found in dirty $\mathrm{MgB}_{2}$ films (large intra-band diffusivity), where large $\lambda$ value ${ }^{\mathrm{xi}}$ and smooth $J_{c}(\mathrm{H})$ dependences ${ }^{\mathrm{xix}}$ (high pinning) have been reported.

\section{Experimental}

The epitaxial $\mathrm{MgB}_{2}$ thin film with the thickness of $310 \mathrm{~nm}$ was grown on a $c$-cut sapphire by the hybrid physical chemical vapor deposition (HPCVD) technique. ${ }^{\text {xi }}$ The $T$ and $\mathbf{H}$ dependence of the magnetization was studied using a superconductor quantum interference device (SQUID) magnetometer. A direct penetration depth $(\lambda)$ measurement at $4 \mathrm{~K}$ was performed by magnetic force microscopy (MFM) based on a direct comparison of the Meisnner response forces between the 
sample and a $\mathrm{Nb}$ reference in situ. ${ }^{\text {xii }}$ The critical currents densities $\left(J_{c}\right)$ were estimated applying the Bean critical-state model to the magnetization data, obtained from hysteresis loops,

$J_{c}=\frac{20 \Delta M}{t w^{2}(l-w / 3)}$, where $\Delta M$ is the difference in magnetization between the top and bottom

branches of the hysteresis loop, and $t, \mathrm{w}$, and $l$ are the thickness, width, and length of the sample $(l>$ $w)$, respectively. The creep measurement $\left[J_{c}(t)\right]$ was recorded over a time period of one hour. The initial time was adjusted considering the best correlation factor in the log-log fitting of the $J_{c}(t)$ dependence. The initial critical state for each creep measurement was prepared by applying a field of $H \sim 4 H^{*}$, where $H^{*}$ is the field for the full-flux penetration. ${ }^{\text {xiii }}$ Electrical resistivity was measured using the standard four-probe technique. The samples were mounted in a rotatable probe and the measurements were performed in applied magnetic fields of between 0 and $9 \mathrm{~T}$. The angular dependence $J_{c}(\theta)$ was measured from the current - voltage (I-V) curve by using the $1 \mu \mathrm{V}$ criteria. Transport measurements were conducted with applied current $(\mathbf{J})$ perpendicular to $\mathbf{H}$ in a maximum Lorentz force configuration. The angle $\theta$ is defined between the applied field and the $c$-axis of the $\mathrm{MgB}_{2}$ (perpendicular to the surface).

\section{Results and discussion}

The $\mathrm{MgB}_{2}$ film superconducting critical temperature $\left(T_{c}\right)$ and its transition width of $T_{c}=39.7$ $\mathrm{K}$ and $\Delta T_{c}=0.1 \mathrm{~K}$, respectively. The residual resistance ratio $\left(\rho^{300 \mathrm{~K}} / \rho^{42 \mathrm{~K}}\right)$ is $\approx 18$. Figure 1 (a) shows the temperature dependence of the upper critical field $\left(H_{c 2}\right)$ and the irreversibility line $\left(H_{i r r}\right)$ with the magnetic field $(\mathbf{H})$ parallel $(/ /)$ and perpendicular $(\perp)$ to the $c$-axis of the sample between $T_{c}$ and $25 \mathrm{~K}$. Below $T \approx 25 \mathrm{~K}, T$ onset $\left(T^{o n}\right)$ and $T$ of zero $\left(T^{\text {zero }}\right)$ resistance are affected by the surface superconductivity, and depend on the applied electrical current density. ${ }^{\text {xiv, } x \mathrm{xv}}$ The $H_{c 2}$ values obtained from magnetic hysteresis loops (not shown) and the $H_{c 2}$ at $20 \mathrm{~K}$ obtained from I-V curves are also included on the graph. Extrapolating to $T=0 \mathrm{~K}$, we obtain $H_{c 2}(0)$ of about $3.5 \mathrm{~T}$, which is close to values found in clean single crystals. ${ }^{\text {xxvi }}$ Using this value, we obtain $\xi_{a b}(0)=10 \mathrm{~nm}$ from $H_{c 2}^{c}=\Phi_{0} /\left[2 \pi \xi_{a b}^{2}(0)\right]$. Fig. 1(b) shows the results of the $H_{c 2}(\theta)$ measurements at $35 \mathrm{~K}$, using the $T^{\text {zero }}$ criteria when the voltage drops to zero, ${ }^{\text {xiv }}$ and the corresponding fit to the effective mass description $H_{c 2}(T, \Theta)=H_{c 2}(T, \Theta=0) \varepsilon(\Theta)$, where $\varepsilon(\Theta)=\left[\cos ^{2} \Theta+\gamma^{-2} \sin ^{2} \Theta\right]^{1 / 2}$, where $\Theta$ is the angle between the applied magnetic field $\mathbf{H}$ and the crystallographic $c$-axis and $\gamma$ is the anisotropy of the critical field. The cusp-like behavior of the experimental data when the field is close to being parallel to the surface (around $\Theta=90^{\circ}$ ) and it can be associated with surface superconductivity. ${ }^{\text {xxiv }}$ Considering that surface superconductivity produces a field enhancement of $H_{c 3}=1.69 H_{c 2}$, ${ }^{\text {xxvii }}$ the $\gamma$ $\approx 2.5$ obtained via the fit shows a good agreement with anisotropy values obtained in clean single crystals. ${ }^{\text {xiv }}$ Analyzing of the data at $T=24 \mathrm{~K}$ (not shown) with the same $T^{\text {zero }}$ criteria, we obtain $\gamma$ $\approx 4.5$. Although the two-band effects result in deviations from the effective mass description, the obtained values of $\gamma$ are in good agreement with those reported in clean systems, ${ }^{\text {xiv }}$ when $H_{c 3}$ effect is taken in to account. It is important to note that the $H_{c 2}(T)$ and $\gamma(T)$ dependences found here are different from those reported in dirty thin films. In that case, the films exhibit large values of $H_{c 2}$ as well as changes in $\gamma$ from 1 to 2 with decreasing temperature. ${ }^{\text {xi,xxviii }}$

Figure 2(a) shows superconducting vortices in the $\mathrm{MgB}_{2}$ film resolved by MFM as bright spots, a result of a repulsive interaction between the tip and the vortices, with magnetization antiparallel to each other. The dark spots in Fig. 2(a) are not anti-vortices since their shapes are irregular compared to the vortices. Instead, they represent nanoscale-sized local inhomogeneities. An inhomogeneous arrangement of vortices similar to the one shown in Fig. 2(a) has been observed previously in $\mathrm{MgB}_{2}$ single crystals, ${ }^{\text {xix }}$ where a nonmonotonic vortex-vortex interaction was considered. This data can be described by changes in the surface energy in analogy with type I and type II superconductors due to two band contributions. ${ }^{\mathrm{xx}}$ However, our data does not allow a 
distinction between effects generated by intrinsic interaction of vortices and irregular pinning due to crystalline defects. Figures 2(b) and 2(c) show single vortices obtained from the $\mathrm{MgB}_{2}$ and a $\mathrm{Nb}$ film reference with the same experimental condition in a single cool-down. Their line profiles along dotted lines in each image are shown in Fig. 2(d). ${ }^{\text {xii }}$ The magnetic penetration depth $(\lambda)$ at $4 \mathrm{~K}$ was estimated by the MFM Meissner method, described elsewhere, ${ }^{x i, x x i i}$ resulting in $\lambda_{\mathrm{ab}}=50 \pm 10 \mathrm{~nm}$.

This value is in agreement with those reported in clean $\mathrm{MgB}_{2}$ samples. ${ }^{\mathrm{x} x x \mathrm{x}}$ Measurements of $J_{c}$ at 5 $\mathrm{K}$ in $\mathrm{MgB}_{2}$ nanobridges of $150 \mathrm{~nm}$ in width, obtained from similar quality of thin films, show $J_{c} \approx$ $160 \mathrm{MAcm}^{-2}$. ${ }^{\text {viii }}$ Considering geometrical effects, ${ }^{\text {xxxii }}$ the reported value can be considered as depairing critical current $\left(J_{0}\right)$, obtained in a vortex-free state at low temperatures, for $\pi$-band contribution. ${ }^{\text {xviii,xxvii }}$ At high field the anomalous evolution of $\xi(H)$ and $\lambda(H)$ predicts a lower $J_{0}(0$ $\mathrm{K})$ value for the $\sigma$ band. ${ }^{\text {iv }}$ The theoretical $J_{0}$ without consider two band contributions ${ }^{17}$ can be estimated via the Ginzburg-Landau equation $J_{0}^{G L}=\frac{c H_{c}}{3 \sqrt{6} \pi \lambda}$, where $c$ is the speed of light in vacuum and $H_{c}=\frac{\Phi_{0}}{2 \sqrt{2} \pi \lambda(0) \xi(0)}$ is the thermodynamic critical field. Using $J_{0}=160 \mathrm{MA} \mathrm{cm}^{-2}$ and $\lambda_{\mathrm{ab}}=50 \mathrm{~nm}$, we obtain $\xi_{\mathrm{ab}} \approx 25 \mathrm{~nm}$ and $H_{c} \approx 1800$ Oe. The $H_{c}$ value is close to those obtained from specific heat measurements, attributed to the $\pi$ - band in clean single crystals $(1500 \mathrm{Oe}) .{ }^{\text {xxxii }}$ In addition, using $\lambda_{a b}=50 \mathrm{~nm}$ and $\xi_{a b}=25 \mathrm{~nm}$, we obtain Ginzburg-Landau parameter $k=2$, which is within the range of the reported values in clean single crystals at low field.

Figure 3(a) shows the log-log plot of $J_{c}$ vs $H$ at four different temperatures $(5,10,20$, and 30 $\mathrm{K})$. The self-field of $J_{c}$ at $T=4 \mathrm{~K}$ is $26 \mathrm{MA} \mathrm{cm}^{-2}$, which is about $16 \%$ of the theoretical value of $J_{0} \approx$ $160 \mathrm{MA} \mathrm{cm}^{-2}$. Three clear distinct regimes can be identified in the $\log -\log J_{c}(H)$ plot, similar to those reported on clean $\mathrm{MgB}_{2}$ single crystals. ${ }^{\mathrm{xvi}}$ The first regime is between 0 and $B^{*}$, where $J_{c}(H) \approx$ constant, the second regime is represented by a power-law dependence $\left(J_{c} \propto H^{-\alpha}\right)$, and the third depicts a fast drop of the $J_{c}(H)$. Although this regime has been discussed in cuprates as a single vortex regime, ${ }^{\mathrm{xxiv}} B^{*}(5 \mathrm{~K}) \approx 0.08 \mathrm{~T}$ is in good agreement with a crossover produced by self-field effect which can be estimated as $B^{*}=J_{c} t$, with $t$ the thickness. ${ }^{\mathrm{xxx}}$ In agreement with that, the first regime in the film clearly seen only at low temperatures, whereas that in single crystals remains still up to high temperatures close $T_{c}{ }^{19}$ The second regime $\left(J_{c} \propto \mathrm{H}^{-\alpha}\right)$ can be fit with $\alpha=1$. The $\alpha$ value in cuprates and pnictide superconductors is related to the type of pinning centers, and ranges between 0.6 and 0.2 depending on their geometry. ${ }^{\text {xxiv }}$ In cuprates an $\alpha=1$ value is well described by the theory for strong pinning, when the vortex excursion driven by thermal fluctuations is comparable to the inter-vortex distance. ${ }^{\mathrm{xxiv}}$ However, high pinning energy and low vortex fluctuations in $\mathrm{MgB}_{2}$ require a different analysis. ${ }^{\mathrm{xix}}$ In this context, dirty $\mathrm{MgB}_{2}$ films, presenting lower self-field $J_{c}$ values at low temperatures but technologically more favorable $J_{c}(H)$ dependences. ${ }^{\text {xix,xxxvi }}$ This fact can be associated with a high density of strong pinning centers, ${ }^{\text {xxvii }}$ and also with a more isotropic behavior produced by large intra-band scattering. ${ }^{\mathrm{xi}}$ In addition, large intra-band scattering increases $\lambda$, and the physics bears similarity to that of single band materials, which reduces $J_{0}$ and affects the pinning energy as discussed below. In this context we analyze the pinning landscape in conjunction with fundamental superconducting properties in our film. Figure 3(b) shows the angular dependence of $J_{c}$ at $35 \mathrm{~K}$ at different $H$. The measurements can be understood via anisotropic scaling of the $J_{c}(\theta)$, however, some clear features, associated with different type of strong pinning centers (such as local inhomogeneities in Fig. 2 a)), are evident. The small peak at $\mu_{0} H<0.3 \mathrm{~T}$ when $\mathbf{H} / / c$-axis represents the presence of correlated disorder, ${ }^{\text {xxxviii }}$ whereas the small shoulder at $\theta \approx 50^{\circ}$ (see inset) can be associated with the pinning by small $\mathrm{MgO}$ precipitates, ${ }^{\mathrm{xxxix}}$ as occurs in YBCO films when pinning is dominated by nanoparticles. ${ }^{\mathrm{xl}}$ The peak effect by small nanoparticles is more pronounced at $T=5 \mathrm{~K}$ and high $H$ (not shown). In order to understand the pinning nature, we analyzed a field dependence of the pinning force $F_{P}=J_{c} H$. When the same type of pinning mechanism dominates over a certain temperature range, the $F_{p}(H, T)$ can be scaled as follows: $F_{p} / F_{p}^{\max } \propto h^{m}(1-h)^{l}, m$ and $l$ are exponents that depend on the pinning 
mechanism, and $h=H / H_{\mathrm{c} 2}(T){ }^{\mathrm{xli}}$ Figure 4 shows the $F_{p} / F_{p}^{\max }$ versus $h$ at different temperatures. The curves for different temperatures show a similar behavior. A peak is present at small $h$, which could be associated with $B^{*}$, and a second broad peak with maximum around $h \approx 0.2$.xvi In spite of deviations at low field, the curves at different temperatures can be scaled with $m=0.5$ and $l=2$ (see Fig. 4), showing a maximum value around $h=0.2$, which can be associated with normal surface pinning as expected in conventional single band superconductors. ${ }^{\text {xli }}$ A similar temperature independent scaling indicates that the pinning mechanisms are temperature independent in the analyzed range. On the other hand, similar pinning mechanism dominates the vortex dynamics in clean $\mathrm{MgB}_{2}$ samples, where a strong suppression of $J_{c}$ with thickness has been reported. ${ }^{\text {xi }}$ As we shown in Fig. 3 (b), it is clear that the pinning mechanism changes with the magnetic field increased. The pinning at low magnetic field is different from that at high field where large defects such as correlated disorder and nanoparticles play a more important role. Fig. 3(b) is also consistent with low angle grain boundaries, present at low density, being the source of correlated pinning, which is manifested in suppression of the $J_{c}$ peak when $\mathrm{H} / / \mathrm{c}$-axis at $\mu_{0} H=0.3 \mathrm{~T}$. In this scenario, any evolution of $\xi(H)$ and $\lambda(H)$ should modify the pinning energy scales, thus making the relative importance of the pinning mechanisms $H$ dependent. Finally, we discuss the third regime associated with a fast drop of $J_{c}(H)$. The value of $H$, where a fast drop starts at each temperature, is similar to those found in single crystals, ${ }^{\mathrm{xvi}}$ ruling out thickness/ $\lambda$ ratio as the mechanism responsible for the change in the flux creep. The similitude between $J_{c}(H)$ in our film and those found in single crystals, ${ }^{19}$ i.e., a fast drop of $J_{c}$ around $\mu_{0} H=1 \mathrm{~T}$ at $5 \mathrm{~K}$, suggests that the pinning is affected by intrinsic superconducting properties such as suppression of the $\pi$-band, and the low band coupling present in clean samples. iv,ix

Figure 5 shows the temperature dependence of the flux creep rate $S(T)$ for different $H$. The values are very small ${ }^{\mathrm{xix}}$ in comparison with cuprates ${ }^{\text {xxiii }}$ and pnictides. ${ }^{\text {xlii }}$ The $J_{c}(t)$ dependence varies logarithmically according to the simple Anderson-Kim model ${ }^{\mathrm{xliii}}$ as $J(t)=J(0)\left[1-\left(\frac{T}{U_{0}}\right) \ln \left(\frac{t}{t_{0}}\right)\right]$.

The basic concept of the flux creep is that a flux line or a flux bundle can be thermally activated to overcome the pinning energy barrier $U_{0}$. At low temperature $U_{0}$ is independent of $T$, and $S$ should change lineally with temperature: $S=T / U_{0} \cdot{ }^{x x i i i}$ The $U_{0}$ values, derived from the measured values of $S$ at low temperature, are shown in the inset of Fig. 5. Although our data can be described well with $U_{0}=A H^{-0.55}$, the analysis of the $U_{0}$ in a wide range of $H$ in $\mathrm{MgB}_{2}$ shows a good correlation with $U_{0}=A B^{\gamma}\left(1-\frac{B}{B^{*}}\right)^{\delta}$. xix Neglecting the parabolic suppression, our fit results in $A \approx 3600$, which is higher than those reported in dirty films, ${ }^{x i x}$ and indicates that beyond the presence of strong pinning centers, $U_{0}$ depends on the intrinsic superconducting properties. A rough estimate of the pinning energy, considering the condensation energy within a coherence volume, ${ }^{24} U_{0}=\left(H_{c}{ }^{2} / 8 \pi\right) 4 / 3 \pi \xi^{3}$, shows that the value is strongly dependent upon both $\lambda$ and $\xi$, which gives rise to $H$ dependence of $J_{c}$ in clean $\mathrm{MgB}_{2} .{ }^{\text {vii }}$ Using our previous estimates of $H_{c} \approx 1800$ Oe and $\xi \approx 25 \mathrm{~nm}$, we obtain $U_{0} \approx$ $60000 \mathrm{~K}$.

Our results show that clean $\mathrm{MgB}_{2}$ films present very high $J_{c}$ values at small magnetic fields and two distinctive different regimes in $J_{c}(H)$. One is for $\mu_{0} H<1 \mathrm{~T}$, which is associated with the suppression of the $\pi$-band. The other is for fields above $1 \mathrm{~T}$, which is associated with more anisotropic $\sigma$-band. ${ }^{i i, i v}$ Large vortex fluctuations ${ }^{x \text { liv }}$ and vortex dissipation ${ }^{\mathrm{xlv}}$ have been reported in clean systems at high fields, which is in agreement with a clear change on the vortex dynamics. The contribution of each band to the $J_{c}$ value depends on inter- and intra-band coupling, density of states 
at the Fermi surface, and Fermi velocity for each band. By considering appropriate parameters a small contribution $(\approx 10 \%)$ of the $\pi$-band to the $J_{c}$ values is expected. ${ }^{x i v}$ This contribution is enhanced by the modification of the parameter, $i$. e. Fermi velocity ratio. The higher $J_{c}(H=0)$ values in clean film (in comparison with dirty samples) can be associated with both higher $J_{0}$ and $U_{0}$ values, which suggests a large $\pi$-band contribution below 1 T. On the other hand, the extrapolation of $U_{0}(H)$ at high magnetic fields suggest that many of the features of the vortex dynamics in the range dominated by the $\sigma$-band in clean $\mathrm{MgB}_{2}$ samples should be similar to those found in pnictides $^{\text {xlii }}$ and HTS cuprates. ${ }^{x x i i}$ In these systems, the combination of high $\kappa$ and $\gamma$ values reduce the pinning energy $U_{0}$ and the creep rate is higher than in conventional low- $T_{c}$ superconductors. The resulting pinning is strongly dependent of the presence of strong pinning centers. ${ }^{\text {xlii }}$ In agreement with that, the angular dependences of $J_{c}$ at different $H$ show that pinning at low magnetic field is different from that at high field where the role of large defects (correlated disorder and nanoparticles) is clearly played.

\section{Conclusions}

We studied intrinsic superconducting properties of a clean $\mathrm{MgB}_{2}$ thin film. We found $\lambda(4 \mathrm{~K})=50 \pm$ $10 \mathrm{~nm}$ and $\xi_{\mathrm{ab}}=10 \mathrm{~nm}$, which results from the effect of the $\pi$-band (low field) and $\sigma$-band (high field) on superconductivity. The $\gamma_{H_{c 2}}$ ranges from 2 close to $T_{c}$ to 4.5 at low temperatures. We found high values of self-field $J_{c}$, that are strongly reduced by $H$, which can be attributed to the suppression of the $\pi$-band. The temperature dependence of the creep rate $S(T)$ is consistent with a simple Anderson-Kim mechanism (huge $U_{0}$ values). Our findings show that different field dependence of the gaps in a multiband superconductor play an important role in defining its vortex dynamics.

\section{Acknowledgments}

This work was supported by the U.S. Department of Energy, Office of Basic Energy Sciences, Division of Materials Sciences and Engineering, and was supported by Institute for Basic Science by Project Code (IBS-R015-D1). N. H. is a member of CONICET (Argentina).

\section{References}

['] J. Nagamatsu et al. Nature London 410 (2001) 63.

[ii] M. Zehetmayer, Supercond. Sci. Technol. 26 (2013) 43001

[iii] N. Hakim, C. Kusko, S. Sridhar, A. Soukiassian, X. H. Zeng, X. X. Xi, Appl. Phys. Lett. 81 (2002) 3603.

$\left.{ }^{[\mathrm{i}}\right]$ M. Eisterer Supercond. Sci. Technol. 20 (2007) 47.

["] X. X. Xi, Rep. Prog. Phys. 71 (2008) 116501.

[ $\left.{ }^{\mathrm{vi}}\right]$ L. Lyard et. al., Supercond. Sci. Technol. 16 (2003) 193.

$\left[{ }^{\mathrm{vii}}\right]$ F. F. Bouquet, Y. Y. Wang, I. I. Sheikin, T. T. Plackowski, A.A. Junod, S. S. Lee, S. S. Tajima, Phys. Rev. Lett. 89 (2002) 257001.

[نiii] M. R. Eskildsen et al., Phys. Rev. Lett. 89 (2002) 187003.

$\left[{ }^{\mathrm{ix}}\right]$ M. Zehetmayer, M. Eisterer, J. Jun, S. M. Kazakov, J. Karpinski, H. W. Weber, Phys. Rev. B 70 (2004) 214516.

[ ${ }^{\mathrm{X}}$ ] T. Klein, L. Lyard, J. Marcus, Z. Holanova, C. Marcenat, Phys. Rev B 73 (2006) 184513.

$\left[{ }^{\mathrm{xi}}\right]$ Jeehoon Kim et al. Phys. Rev. B 86 (2012) 024501. 
[ $\left.{ }^{\mathrm{xii}}\right]$ L. Komendova, Yajiang Chen, A. A. Shanenko, M.V. Milosevic, and F. M. Peeters. Phys. Rev Lett 108, (2012) 207002.

[ $\left.{ }^{\mathrm{xiii}}\right]$ Elias Galan, Daniel Cunnane, X. X. Xi, Ke Chen. Supercond. Sci. Technol. 27 (2014) 65015.

[ $\left.{ }^{\mathrm{xiv}}\right]$ E. J. Nicol, J. P Carbotte. Phys. Rev. B 72 (2005) 14520.

$\left[{ }^{\mathrm{xv}}\right]$ Sergey Lee, T. Masui, H. Mori, Yu Eltsev, A. Yamamoto, S. Tajima, Supercond. Sci. Technol. 16 (2003) 213.

$\left[{ }^{\mathrm{xvi}}\right]$ Z. X. Shi et al. Phys. Rev. B 68 (2003) 104514.

$\left[{ }^{\text {xvii }}\right.$ Hyeong-Jin Kim, W. N. Kang, Eun-Mi Choi, Mun-Seog Kim, Kijoon H. P. Kim, Sung-Ik Lee, Phys. Rev Lett 87 (2001) 087002.

[xviii] C. G. Zhuang et al. J. Appl. Phys. 104 (2008) 013924.

$\left[{ }^{\mathrm{xix}}\right]$ J. R. Thompson, K. D. Sorge, C. Cantoni, H. R. Kerchner, D. K. Christen, M. Paranthaman, Supercond. Sci. Technol. 18 (2005) 970.

$\left[{ }^{\mathrm{xx}}\right]$ G. Blatter, M. V. Feigel'Man, V. B. Geshkenbein, A. I. Larkin, V. M. Vinokur, Rev. Mod. Phys. 66 (1994) 1125.

$\left[{ }^{x \times i}\right]$ A. V. Pogrebnyakov, J. M. Redwing, J. E. Jones, X. X. Xi, S. Y. Xu, Q. Li, V. Vaithyanathan, D. G. Schlom, Appl. Phys. Lett. 82 (2003) 4319.

$\left[{ }^{\mathrm{xxii}}\right]$ Jeehoon Kim et al. Supercond. Sci. Technol. 25 (2012) 112001.

[ $\left.{ }^{x x i i i}\right]$ Y. Yeshurun, A. P. Malozemoff, A. Shaulov Rev. Mod. Phys. 68 (1996) 911.

[xiv] A. Rydh et al. Phys. Rev 68 (2003) 172502.

$\left.{ }^{\mathrm{xxv}}\right]$ U. Welp et al. Phys. Rev. B 67 (2003) 012505.

$\left.{ }^{[x x v i}\right]$ L. Lyard et al. Supercond. Sci. Technol. 16 (2003) 193.

[ $\left.{ }^{x x v i i}\right]$ M Thinkam, Introduction to superconductivity (McGraw Hill, New York, 1996).

[ $\left.{ }^{\text {xxviii }}\right]$ Satoru Noguchi et al. Supercond. Sci. Technol. 22, 055004 (2009)

${ }^{[\mathrm{xxix}}$ ] Victor Moshchalkov, Mariela Menghini, T. Nishio, Q. H. Chen, A.V. Silhanek, V. H. Dao, L. F. Chibotaru,1 N. D. Zhigadlo, and J. Karpinski. Phys. Rev Lett 102, 117001 (2009)

$\left[{ }^{\mathrm{xx}}\right]$ A. Chaves, L. Komendova, M. V. Milosevic, J. S. Andrade Jr., G. A. Farias, and F. M. Peeters. Phys. Rev B 83, 214523 (2011).

$\left[{ }^{\mathrm{xxxi}}\right]$ Daniel Cunnane, Chenggang Zhuang, Ke Chen, X. X. Xi, Jie Yong, T. R. Lemberger, Appl. Phys. Lett 102 (2013) 072603.

[ ${ }^{\mathrm{xxxii}]}$ B. J. Yuan, J. P. Whitehead, Phys. C 231 (1994) 395.

$\left[{ }^{\mathrm{xx} x i i i}\right] \mathrm{T}$. Klein (private communication)

[ $\left.{ }^{\mathrm{xxxiv}}\right]$ C. J. van der Beek et al. Phys. Rev. B 66 (2002) 024523.

$\left[{ }^{\mathrm{xxx}}\right]$ N. Haberkorn, M. Miura, B. Maiorov, G. F. Chen, W. Yu, L. Civale, Phys. Rev. B 84 (2011) 94522.

$\left[{ }^{\mathrm{xxxvi}}\right]$ A film similar to the analyzed in reference 14 , has self-field $J c(10 \mathrm{~K})=6.8 \mathrm{MAcm}-2$, and $J c \alpha \mathrm{H}^{-\alpha}$ dependences well described by $\alpha=0.3$.

$\left[{ }^{\mathrm{xxxvii}}\right]$ L. Gu, B. H. Moeckly, D. J. Smith, J. Cryst. Growth 280 (2005) 602.

[ $\left.{ }^{\mathrm{xxxviii}}\right]$ L Civale et. al. Appl. Phys. Lett 84 (2004) 2121.

$\left[{ }^{\mathrm{xxxix}}\right]$ Y. Zhu et al. Supercond. Sci. Technol. 23 (2010) 095008.

[ $\left.{ }^{\mathrm{xl}}\right]$ V. F. Solovyov et al., 2007 Supercond. Sci. Technol. 20 (2007) 20.

$\left[{ }^{\mathrm{xl}}\right]$ D. Dew-Hughes, Philos. Mag. 30 (1974) 293.

[xlii] T. Tamegai et. al. Supercond. Sci. Technol. 25 (2012) 84008.

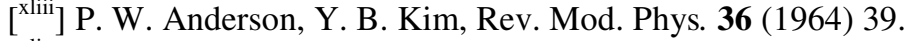

$\left[{ }^{\text {xliv }}\right.$ ] T. Masui et al. Physica C 383 (2003) 299.

$\left[{ }^{\mathrm{xlv}}\right.$ ] Yu Eltsev et al. Phys Rev B 65 (2002) 140501.

Figure 1. (a) Temperature dependence of the upper critical field $\left(H_{c 2}\right)$ and the irreversibility line $\left(H_{i r r}\right)$ in a $\mathrm{MgB}_{2}$ thin film. (b) Angular dependence of $H_{i r r}$ at $35 \mathrm{~K}$ and fit by using the anisotropic mass equation described in the text.

Figure 2. (a) Large field of view image of a $\mathrm{MgB}_{2}$ film. Bright spots are vortices. Dark spots represent local inhomogeneities in the film. (b)-(c) show a single vortex image of $\mathrm{MgB}_{2}$ and $\mathrm{Nb}$, respectively. (d) MFM profiles (shift of the resonance frequency of a magnetic cantilever) of the vortices along the dotted lines in (b) and (c). The lift height of the probe tip is $300 \mathrm{~nm}$. 
Figure 3. (a) Critical current density $\left(J_{c}\right)$ vs. magnetic field $(H)$ at different temperatures obtained by using the Bean model. (b) Angular dependence of the critical current density $J_{c}(\theta)$ at $35 \mathrm{~K}$ in different applied magnetic fields $\left(\mu_{0} H=0.05,0.1,0.2\right.$ and $\left.0.3 \mathrm{~T}\right)$. Inset: $J_{c}(\theta)$ at $20 \mathrm{~K}$ and $\mu_{\mathrm{o}} H=1$ T. Arrow indicates the peak of $J_{c}$ at $\theta \approx 50^{\circ}$.

Figure 4. Normalized pinning force $\left(F_{p}\right)$ versus normalized magnetic field $\left[h=H / H_{i r r}(T)\right]$ at different temperatures.

Figure 5. Temperature $(T)$ dependences of the creep rate $S=-\frac{d\left(\ln J_{c}\right)}{d(\ln t)}$ at different applied magnetic fields. Inset: Pinning energy was obtained from $S=T / U_{0}$.
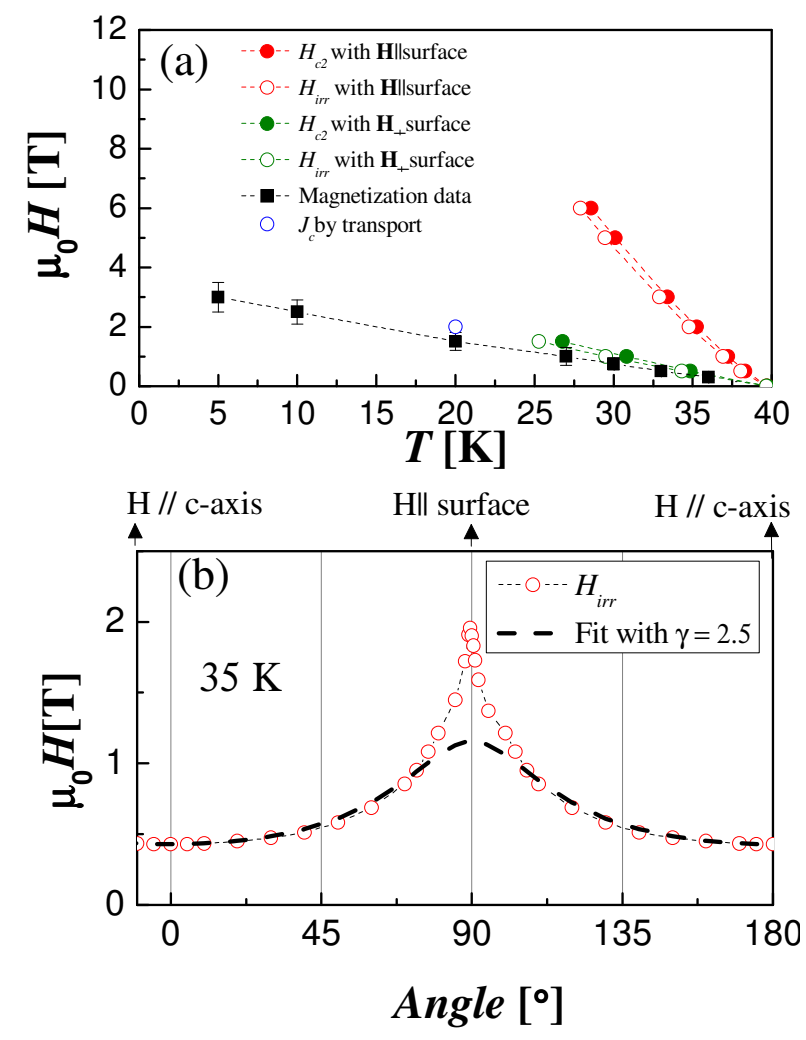

Figure 1 

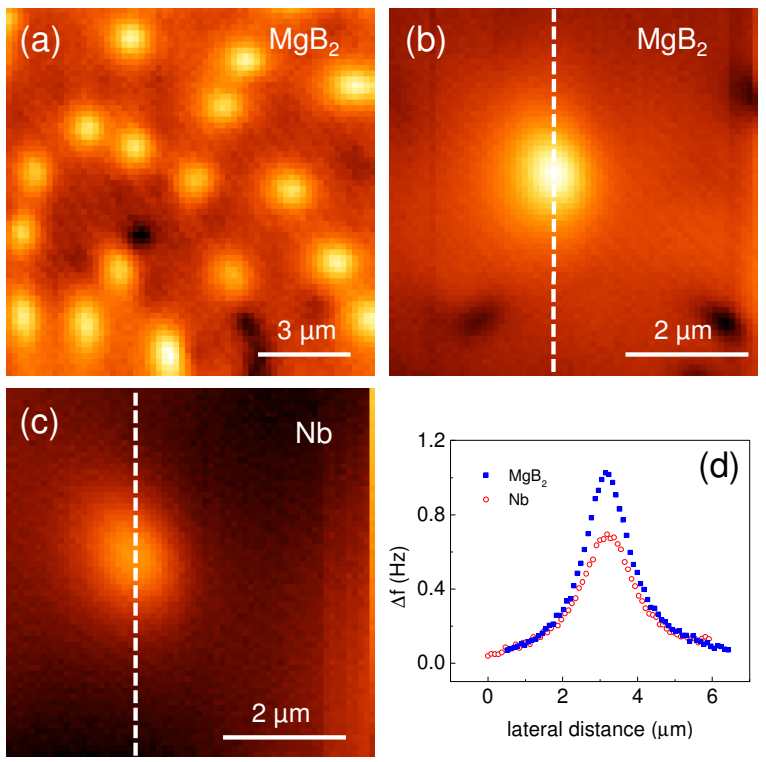

Figure 2
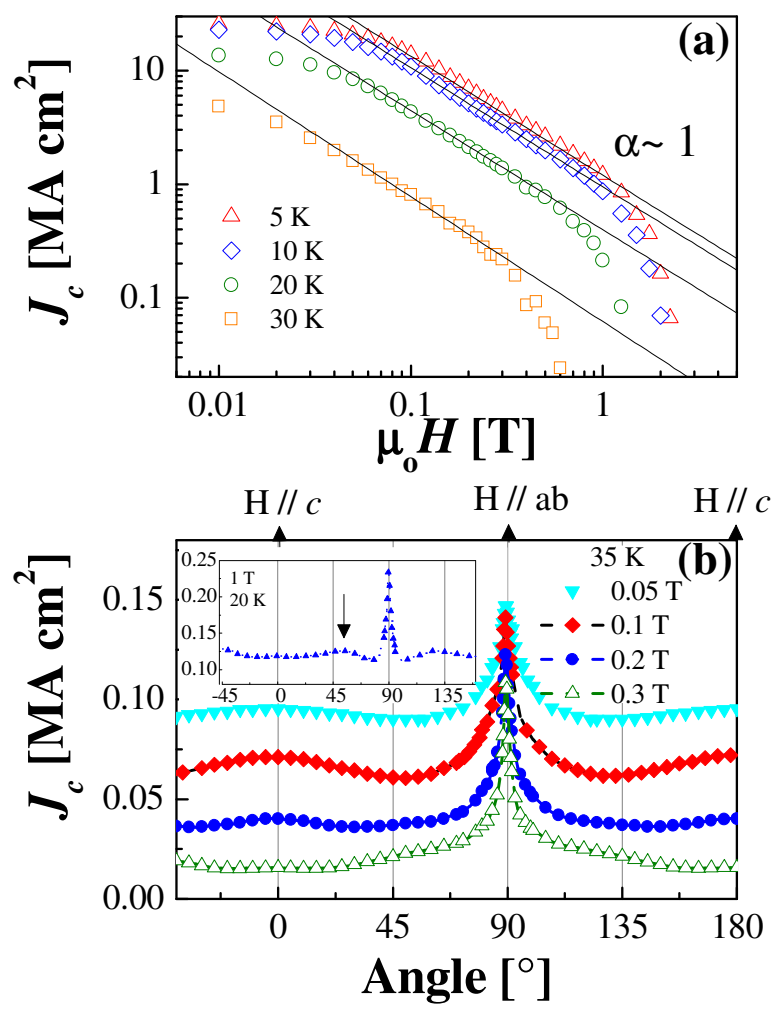

Figure 3 


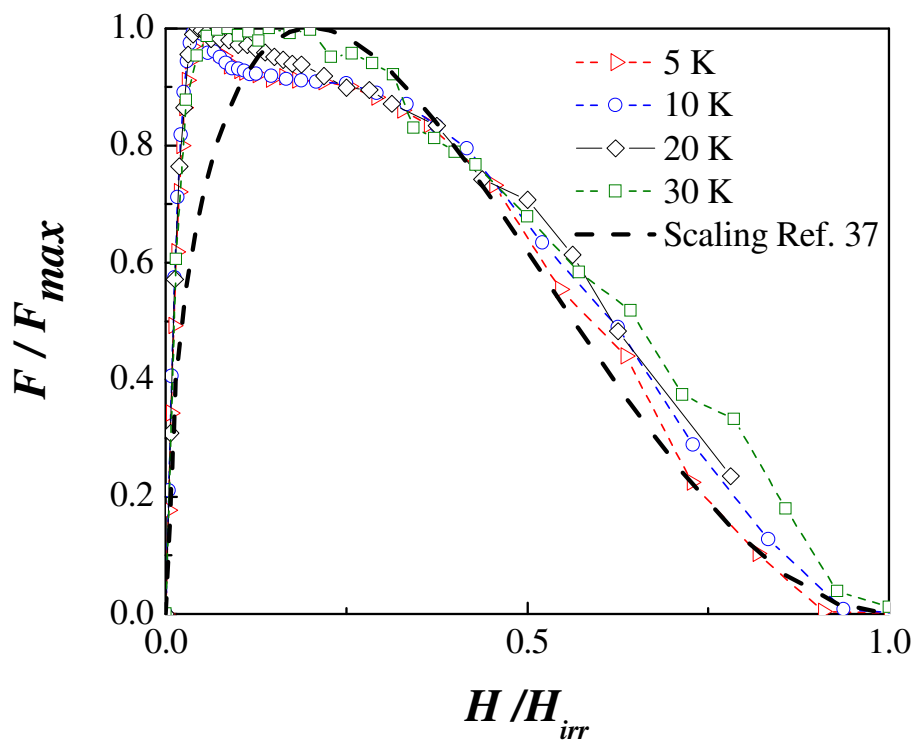

Figure 4

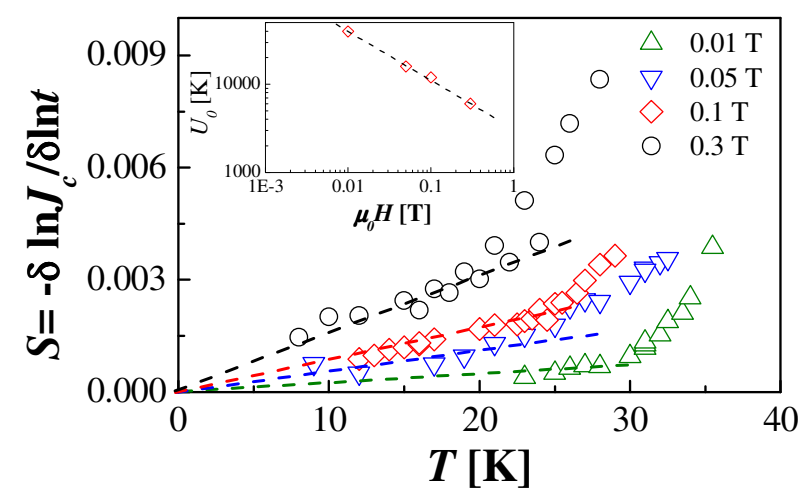

Figure 5 